\title{
Cushing's syndrome due to adrenocorticotropic hormone-secreting metastatic neuroendocrine tumor of unknown primary origin: a case report and literature review
}

\author{
Hayri Bostan ${ }^{1}$ (D Hakan Duger ${ }^{1} \cdot$ Pinar Akhanli $^{1} \cdot$ Murat Calapkulu$^{1} \cdot$ Tugba Taskin Turkmenoglu $^{2}$. \\ Ayse Kevser Erdol ${ }^{3}$. Serap Akcali Duru ${ }^{3} \cdot$ Muhammed Erkam Sencar ${ }^{1} \cdot$ Muhammed Kizilgul $^{1} \cdot$ Bekir Ucan $^{1}$. \\ Mustafa Ozbek ${ }^{1} \cdot$ Erman Cakal $^{1}$
}

Received: 15 May 2021 / Accepted: 24 August 2021 / Published online: 3 September 2021

(c) Hellenic Endocrine Society 2021

\begin{abstract}
Background In this article, we present a case of neuroendocrine neoplasm of unknown primary origin (UPO NEN), which is a rare cause of ectopic Cushing's syndrome (ECS) presenting numerous challenges, together with a literature review. Case report A 43-year-old male patient presented with clinical features consistent with Cushing's syndrome (CS) and adrenocorticotropic hormone (ACTH)-dependent hypercortisolemia. Despite a suspicious lesion on pituitary MRI, the high-dose dexamethasone suppression test and bilateral inferior petrosal sinus sampling results were not compatible with Cushing's disease. Bilateral non-homogeneous opacities were observed in the thorax CT of the patient, who also had a history of COVID-19 infection, but no tumoral lesion was detected. When ${ }^{68} \mathrm{Ga}$-SSTR PET/CT and ${ }^{18}$ FDG-PET/CT were performed, multiple metastatic foci were detected in mediastinal and hilar lymph nodes and the axial skeleton. Paratrachealsubcarinal lymph nodes were excised mediastinoscopically, and the diagnosis of NEN was made. Histopathological findings indicated that the possible origin was an atypical pulmonary carcinoid with a low Ki-67 labeling index. After controlling hypercortisolemia, a regimen of somatostatin analogs and capecitabine plus temozolomide was decided upon as treatment by a multidisciplinary council.

Conclusion This is a challenging case of UPO NEN presenting with ECS and confounding factors, such as previous infection and incidental lesions, during the diagnosis process. The case in question highlighted the fact that atypical pulmonary carcinoid with a low proliferation index may cause visible metastases even when radiologically undetectable.
\end{abstract}

Keywords Ectopic Cushing's syndrome $\cdot$ Neuroendocrine neoplasm $\cdot$ Unknown primary origin $\cdot$ Ga-68 labeled somatostatin receptor PET/CT · Atypical pulmonary carcinoid $\cdot$ Temozolomide

\section{Introduction}

Hayri Bostan

drhayribostan@gmail.com

1 Department of Endocrinology and Metabolism, University of Health Sciences, Diskapi Yildirim Beyazit Training and Research Hospital, Ziraat Mahallesi, Şht. Ömer Halisdemir Blv. No. 1, 6110 Ankara, Turkey

2 Department of Pathology, University of Health Sciences, Diskapi Yildirim Beyazit Training and Research Hospital, Ankara, Turkey

3 Department of Chest Diseases, University of Health Sciences, Diskapi Yildirim Beyazit Training and Research Hospital, Ankara, Turkey
Ectopic Cushing's syndrome (ECS) is a rare condition which can have serious consequences due to a number of hormonal and tumoral aspects. Approximately $4 \%$ of adrenocorticotropic hormone (ACTH)-dependent Cushing's syndrome (CS) cases are composed of malignant neuroendocrine neoplasms (NENs) that secrete ectopic ACTH [1]. Although in the past the most common cause of ECS was considered to be small cell lung carcinoma, in recent years, thanks to increasing awareness, well-differentiated bronchial carcinoids have gained prominence in explaining the etiology [2]. Visible metastasis can be detected at the time of diagnosis in approximately $40-50 \%$ of all NEN cases. The primary origin cannot be determined in $13 \%$ of metastatic NEN cases [3]. 
Failure to determine the primary lesion in metastatic NEN patients with ACTH secretion makes the effort to control both the tumor and the symptoms associated with hypercortisolemia challenging.

In this article, we report a case of metastatic NEN of unknown primary origin, which is a rare cause of ECS and presents various difficulties in the diagnosis process and treatment decision.

\section{Case presentation}

A 43-year-old male patient presented at our clinic in November 2020 with complaints of muscle weakness in both legs, edema on the dorsum of the foot, and weight gain of $10 \mathrm{~kg}$ in the last 6 months. He had a 2-year history of hypertension, as well as obsessive-compulsive disorder and prediabetes for 1 year. In addition, 4 months before presentation, the patient had tested positive for coronavirus disease 2019 (COVID19) infection in July, 2020. Physical examination revealed moon face, buffalo hump, oral candidiasis plaques, central obesity, purple striae over the abdomen, easy bruising, and bilateral pitting edema in the pretibial region and the dorsal region of the foot. There was no history of exogenous steroid use or alcohol consumption.

The patient history and physical examination findings were compatible with CS, and the overnight dexamethasone suppression test was $28.7 \mu \mathrm{g} / \mathrm{dL}$ (reference range $<1.8$ ). Morning serum baseline cortisol was $27.3 \mu \mathrm{g} / \mathrm{dL}$ (reference range: $6.2-19.4$ ), late-night serum cortisol was $26.0 \mu \mathrm{g} / \mathrm{dL}$ (reference range $<7.5$ ), and 24-h urine free cortisol (UFC) was $773.59 \mu \mathrm{g} /$ day (reference range: 4.2-60). Plasma ACTH levels were checked twice and were recorded as 481 and 365 $\mathrm{pg} / \mathrm{mL}$ (reference range: 0-46). Serum cortisol levels were determined with CMIA (chemiluminescent microparticle immunoassay), plasma ACTH levels with ECLIA (enzymelinked chemiluminescent immunosorbent assay), and 24-h UFC levels with LC-MS (lipid chromatography-mass spectrometry) methods. Hypercortisolemia was confirmed, and hypokalemia $\left(\mathrm{K}^{+}: 3.18 \mathrm{mEq} / \mathrm{L}\right.$, reference range: $\left.3.5-5.1\right)$ was also observed. Liver enzyme and kidney function tests were normal. The hormone panel showed the following: thyroid-stimulating hormone (TSH), $0.93 \mathrm{mIU} / \mathrm{L}$ (reference range: $0.27-4.2$ ); free thyroxine (fT4), $0.72 \mathrm{ng} / \mathrm{dL}$ (reference range: $0.93-1.7$ ); follicle-stimulating hormone (FSH), 0.86 IU/L (reference range: 1.5-12.4); luteinizing hormone (LH), $<0.3 \mathrm{IU} / \mathrm{L}$ (reference range: 1.7-8.6); and total testosterone, $55.8 \mathrm{ng} / \mathrm{dL}$ (reference range: $280-800$ ). As an increase in TSH levels was not detected with the thyrotropin-releasing hormone (TRH) stimulation test, it was thought that the cause of hypothyroidism might be impaired TSH response to TRH secondary to hypercortisolemia (Table 1).

Magnetic resonance imaging (MRI) of the pituitary gland was performed, and a suspicious $2 \mathrm{~mm}$ microadenoma was observed on the right side of the pituitary gland in the sagittal planes of the T1-weighted sequence (Fig. 1). Serum cortisol and UFC levels were not suppressed after the highdose dexamethasone suppression test (Table 1). In bilateral inferior petrosal sinus sampling (BIPSS), no gradient was detected between peripheral and central ACTH levels before and after corticotropin-releasing hormone $(\mathrm{CRH})$ infusion (Table 1). In light of dynamic test results, it was speculated that the suspicious lesion in the pituitary gland might be incidental, and the main source could be an ectopic ACTH

Table 1 Dynamic endocrinologic tests for differential diagnosis

\section{${ }^{a}$ TRH stimulation test}

Time (min)

$\begin{array}{lc} & 0 \\ \text { TSH } & 0.12 \\ { }^{\boldsymbol{b}} \text { High-dose dexamethasone suppression test (HDDST) } \\ & \text { Before the test } \\ & (08: 00 \mathrm{AM}) \\ \text { Serum cortisol } & 27.1 \\ \text { 24-h urine free cortisol } & 773.59\end{array}$

${ }^{c}$ Bilateral inferior petrosal sinus sampling (BIPSS)

The time of CRH infusion (min)

$\begin{array}{lllllll} & -5 & 0 & +2 & +5 & +10 & 0-46 \mathrm{pg} / \mathrm{mL} \\ \text { Peripheral ACTH } & 266 & 475 & 418 & 426 & 445 & 316 \\ \text { Right central ACTH } & 246 & 428 & 498 & 435 & 355 & 499 \\ \text { Left central ACTH } & 145 & 492 & 519 & 559\end{array}$

ACTH adrenocorticotropic hormone; $C R H$ human corticotropin-releasing hormone; $T R H$ thyrotropin-releasing hormone; TSH: thyroid-stimulating hormone

${ }^{a}$ TRH stimulation test, ${ }^{b}$ High-dose dexamethasone suppression test (HDDST), ${ }^{c}$ Bilateral inferior petrosal sinus sampling (BIPSS) 


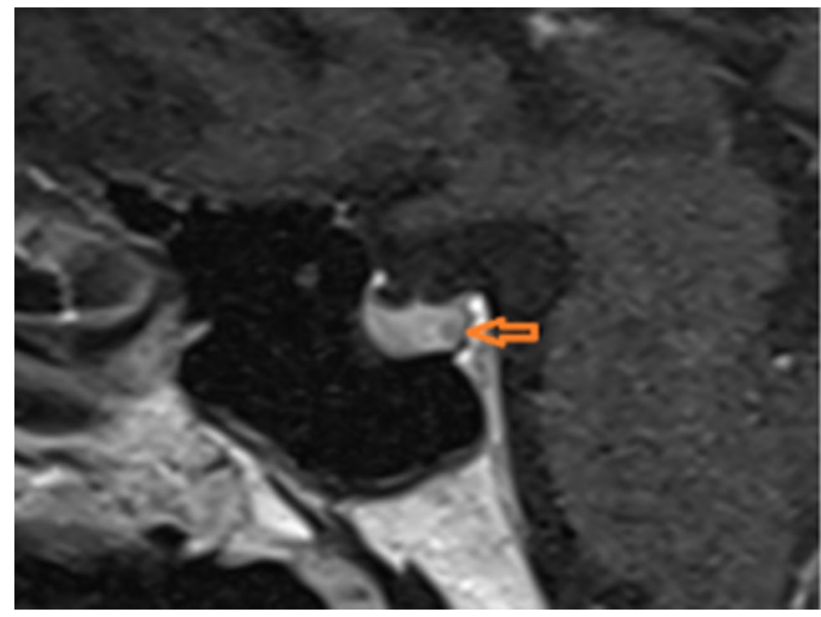

Fig. 1 Pituitary MRI revealed a suspicious microadenoma in the sagittal planes of the T1-weighted sequence (yellow arrow)

secretion. Chromogranin A level measured on high suspicion of NEN was found to be slightly elevated $(237 \mathrm{ng} / \mathrm{mL}$ (reference range: 0-100)). In addition, genetic testing was not considered for multiple endocrine neoplasia type 1 , since the patient had no family history, while his ionized calcium, intact parathormone, and prolactin levels were within normal limits.

A thin-slice whole-body CT was performed in order to detect the ectopic focus in November 2020. Bilateral peripheral non-homogeneous densities were detected on thorax CT (Fig. 2a, b), primarily associated with the recent COVID-19 infection, and no pathology was observed on cervical and abdominal CT. Six weeks after the first imaging, gallium-68 labeled somatostatin receptor PET/CT $\left({ }^{68} \mathrm{Ga}\right.$-SSTR PET/CT $)$ was performed, and increased activity uptake was observed in the paratracheal, subcarinal lymph nodes and in multiple sclerotic lesions in the axial skeleton (Fig. 3a-c). Bronchoscopy was also performed, but no tumor was detected in the trachea or bronchial lumen. ${ }^{18}$ FDG-PET/CT was also applied in order to identify the possible primary origin and to better determine the spread of the aggressive NEN. In ${ }^{18}$ FDG-PET/ CT, as in ${ }^{68} \mathrm{Ga}$-SSTR PET/CT, increased ${ }^{18} \mathrm{FDG}$ uptake was observed in the right paratracheal, right hilar, subcarinal lymph nodes, and axial skeleton, the largest being in the L5 vertebra (Fig. 3d-f). In addition, low-intensity ${ }^{18}$ FDG uptake was detected in the ribs, sternum, iliac bones, and both scapulae, consistent with metastatic disease.

Surgical excision was performed in the right paratracheal and subcarinal lymph nodes using a mediastinoscopic approach. Pathological analyses performed with hematoxylin and eosin (H\&E) staining revealed a tumor composed of monomorphic epithelial cells with round to oval nuclei with salt and pepper chromatin, inconspicuous nucleoli, and moderate to abundant eosinophilic cytoplasm forming nests and trabecular structures intermingled with sparse lymphoid cells (Fig. 4a). Immunohistochemical studies showed diffuse staining with chromogranin and TTF-1, moderate staining with synaptophysin, and sparse staining with CD56 in neoplastic cells (Fig. 4b-d). There was no staining for calcitonin, thyroglobulin, or CDX2. Extensive lymphovascular invasion and two mitoses in $2 \mathrm{~mm}^{2}$ were observed (Fig. 4e),
Fig. 2 Peripheral non-homogeneous densities on the parenchyma of the right lung (a, b) (yellow arrows), primarily associated with COVID-19 infection 4 months ago, have appeared to regress in $\mathrm{CT}$ sequences of functional imaging performed approximately 2 months after the first imaging $(\mathbf{c}, \mathbf{d})$

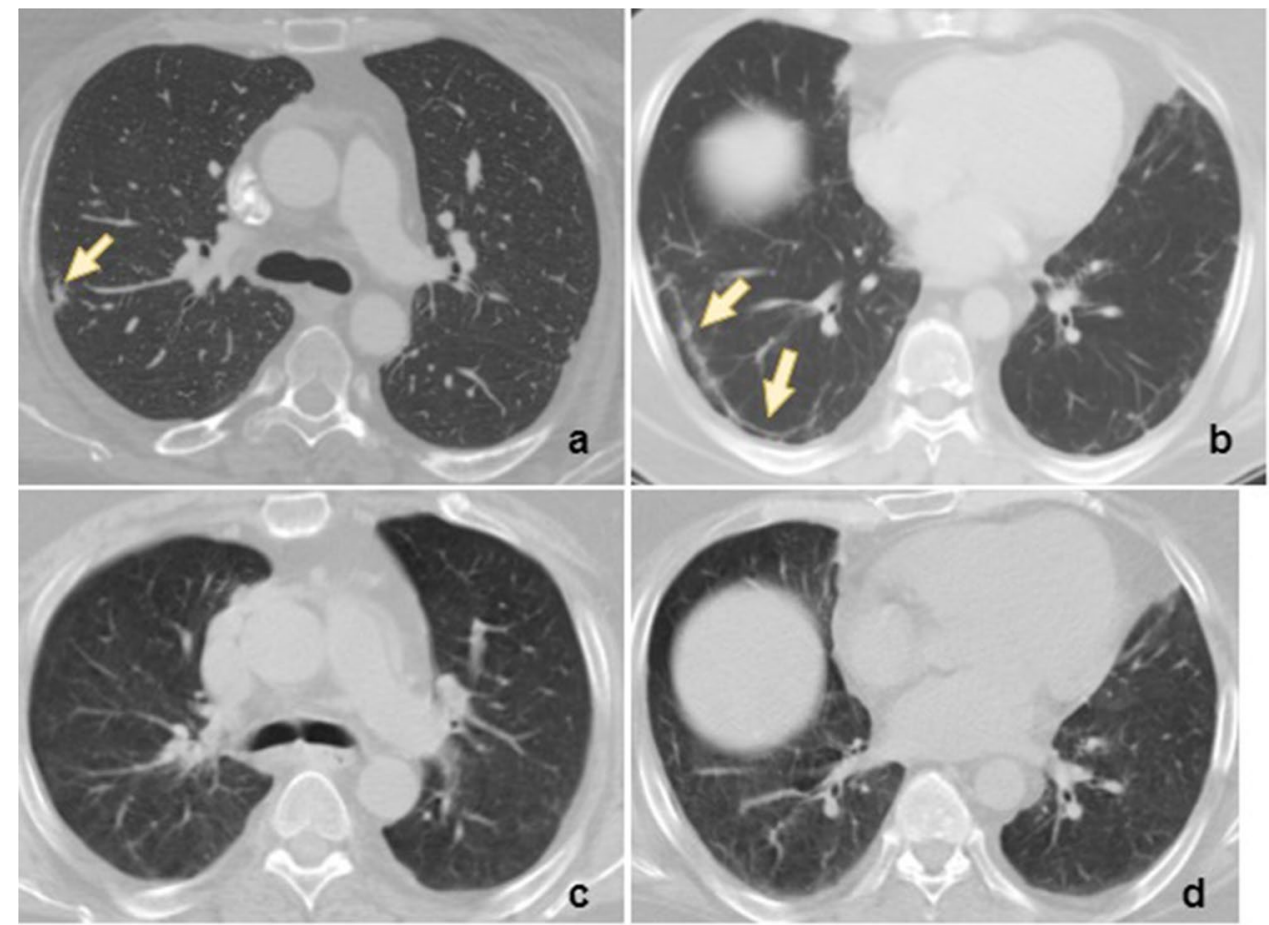




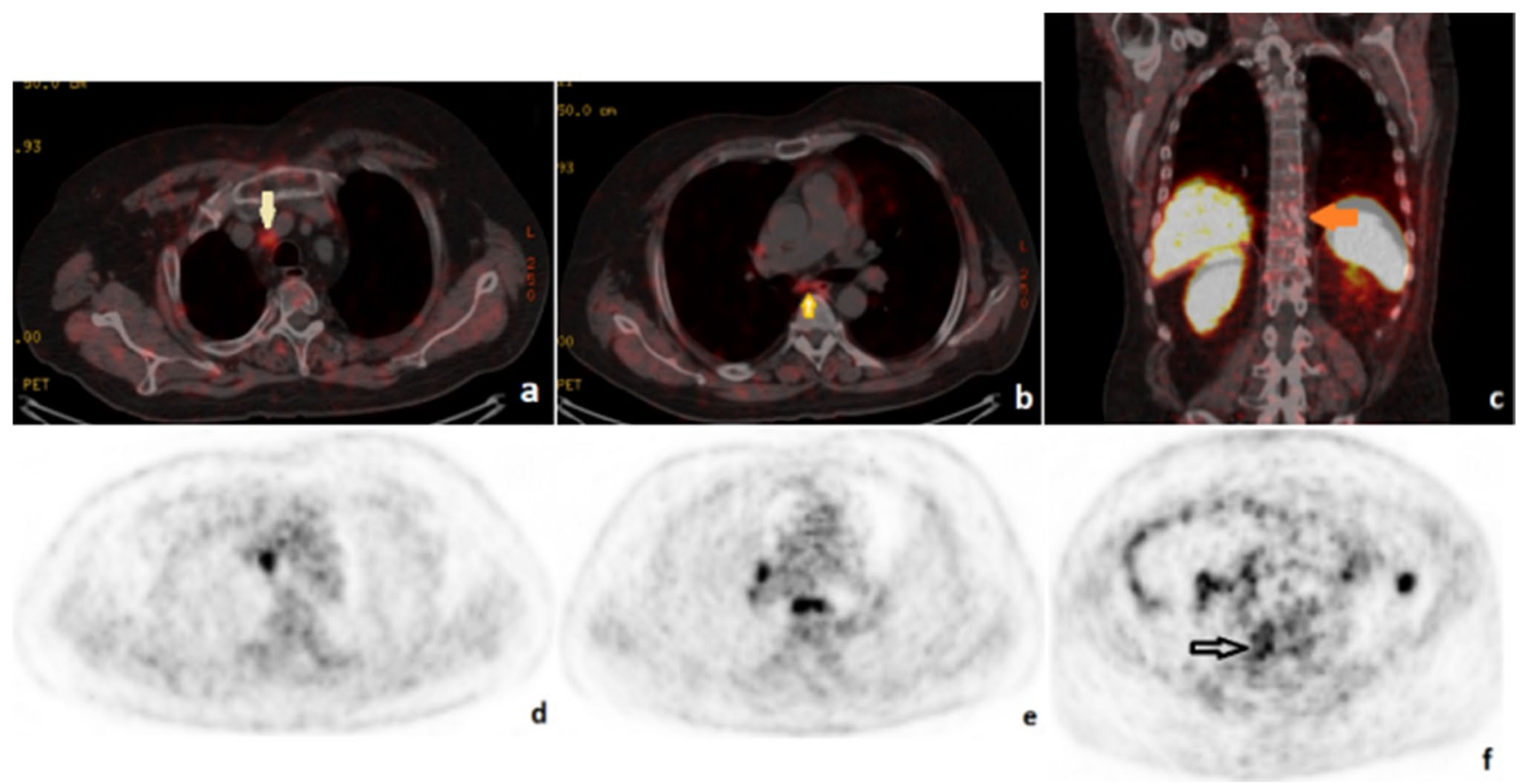

Fig. $3{ }^{68} \mathrm{Ga}$-SSTR PET/CT scan revealed increased uptake in the paratracheal (a), subcarinal lymph nodes (b), and in diffuse sclerotic lesions of the axial skeleton (c) (yellow arrows). In the ${ }^{18}$ FDG-PET/ CT scan, the highest ${ }^{18}$ FDG uptake was detected in the right paratra-

but necrosis was not detected. The Ki-67 labeling index was low (5-8\%) (Fig. 4f). Based on the microscopic and immunohistochemical findings, NEN metastasis was confirmed, and the possible origin of the tumor was presumed to be an atypical pulmonary carcinoid.

Enoxaparin for thromboprophylaxis and trimethoprim/ sulfamethoxazole for prophylaxis of Pneumocystis jirovecii pneumonia were initiated immediately after hospitalization of the patient. To control hypertension and hypokalemia, in addition to ramipril $5 \mathrm{mg}$ treatment, spironolactone $50 \mathrm{mg}$ was given, and oral potassium replacement was administered as needed. Metyrapone was started in order to control hypercortisolemia before second-line imaging methods were used. The initial dose was $1500 \mathrm{mg} /$ day; however, because of an increase in gastrointestinal complaints and the presence of adrenal insufficiency symptoms, the dose was reduced to $500 \mathrm{mg} / \mathrm{day}$, and $20 \mathrm{mg} /$ day hydrocortisone was added to the treatment. UFC levels decreased to $0.59 \mu \mathrm{g} /$ day (reference range 4.2-60) and remained within the normal range during the 7-month follow-up (Fig. 5). After controlling the hypercortisolemia, thyroid function tests and total testosterone levels were normalized in 2 weeks. Considering that the primary origin of the metastatic disease might be a pulmonary atypical carcinoid (AC), a multidisciplinary council decided to start a regimen of temozolomide plus capecitabine (CAPTEM) with lanreotide $90 \mathrm{mg} / \mathrm{month}$. The CAPTEM regimen consisting of temozolomide $360 \mathrm{mg} /$ day cheal (SUVmax: 10.69) (d), right hilar and subcarinal lymph nodes (SUVmax: 9.52) (e), and L5 vertebra (black arrow) (SUVmax: 6.11) (f). SUVmax, maximum standardized uptake value

for 5 days and capecitabine $4500 \mathrm{mg} /$ day for 14 days each cycle was initiated, and meanwhile, the metyrapone treatment was continued. In addition to normalized serum cortisol and UFC levels after the metyrapone treatment, ACTH levels also tended to decrease after the CAPTEM regimen (Fig. 5). Ten cycles of radiotherapy and $4 \mathrm{mg}$ of zoledronic acid were administered due to extensive symptomatic vertebral metastases. Although the ${ }^{18}$ FDG-PET/CT scan was repeated 9 months after the first imaging and 4 months after the initiation of the cytotoxic treatment, the primary focus was still not detected, but a significant decrease was observed in the number, size, and metabolic activity uptake of metastatic foci. Hypercortisolemia and related comorbidities were observed to be under control during this period, and there was no cytotoxic treatment-related toxicity.

\section{Discussion}

Herein, we describe a 43-year-old male patient with metastatic neuroendocrine neoplasm of unknown primary origin (UPO NEN). ECS is a challenging area of endocrine practice that may require lengthy diagnostic journeys to establish the location of the primary lesion.

Although the actual incidence and prevalence of ECS are not known, it constitutes 9-18\% of ACTH-dependent CS cases [1, 2]. It has been reported that ECS occurs in 3.2-6\% 
Fig. 4 Monomorphic epithelial tumor cells forming nests and trabecular structures in the right paratracheal lymph node $(H \& E, \times 200)(\mathbf{a})$, and immunohistochemically (IHC), diffuse chromogranin staining $($ IHC,$\times 100)(\mathbf{b})$, moderate synaptophysin staining (IHC, $\times$ 200) (c), diffuse nuclear TTF-1 staining $(\mathrm{IHC}, \times 100)(\mathbf{d})$ in the tumor cells. Mitotic figure (black arrow) (H\&E, $\times 1000)$ (e) and the low Ki-67 labeling index $(\mathrm{IHC}, \times 400)(\mathbf{f})$ of the neuroendocrine neoplasm are also demonstrated

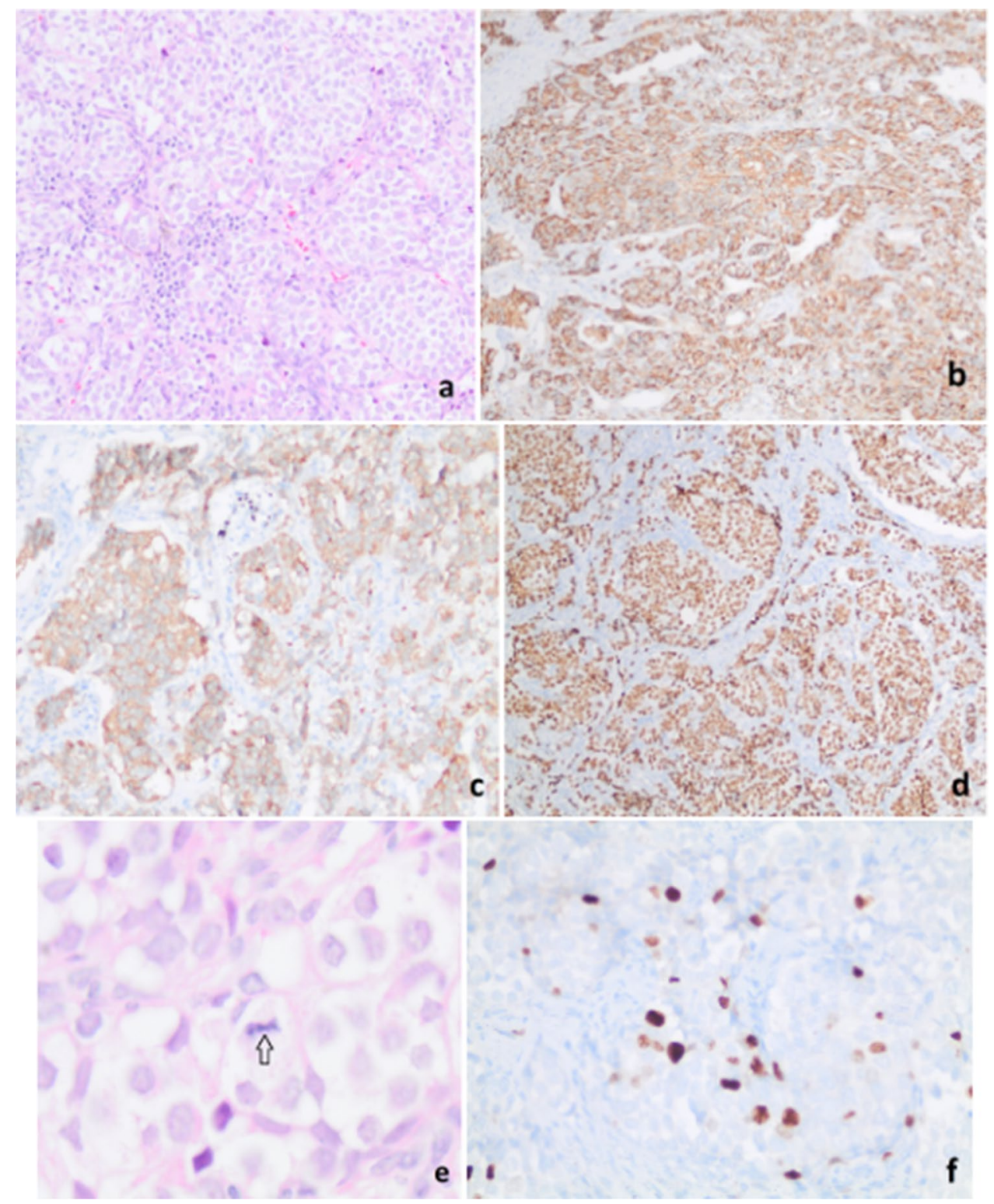

of NENs, and the tumor is often localized in the lung or thyroid in cases with ECS [4-6]. In UPO NENs, which constitute $13 \%$ of metastatic NENs, the most common cause of the functional syndrome is carcinoid syndrome; ACTH secretion can also be seen occasionally [3, 7]. Surgical series have reported that metastases often exist in the liver and peritoneal region, and the primary origin that can be detected intraoperatively is mostly in the small intestine [8-10].

Pituitary MRI is an important guide in the differential diagnosis of CD and ECS, but false-positive or false-negative imaging may be experienced, as in the present case [11]. CRH administration combined with BIPSS is still the "gold standard" method to differentiate ECS from CD [12]. Thin-slice whole-body CT is recommended as the first-line imaging method to detect the tumor focus [13, 14]. Although it is a very effective method for detecting overt tumors, pulmonary embolism and infections can mask small NENs on CT [2]. In the current case, the recent history of COVID19 infection and the presence of multiple non-homogeneous opacities in the lungs may have limited the detection of a possible pulmonary carcinoid at presentation. However, functional imaging performed 2 months later revealed that previously observed non-homogeneous densities had mostly disappeared (Fig. 2c, d).

The superiority of ${ }^{68} \mathrm{Ga}$-SSTR PET/CT over octreoscan in detecting NEN has been demonstrated, while its sensitivity in detecting the primary tumor in occult NEN cases has been reported as $57 \%[2,15]$. Though ${ }^{18} \mathrm{FDG}$-PET/CT is effective in revealing NENs with aggressive behavior, it has limited benefit in defining slow-growing, well-differentiated NENs $[13,16,17]$. In the present case, both functional imaging methods were compatible with metastatic disease, and 
Fig. 5 The graph depicts serum cortisol, 24-h UFC, and ACTH variation following the initiation of metyrapone and CAPTEM regimen. ACTH, adrenocorticotropic hormone; CAPTEM, temozolomide plus capecitabine; 24-h UFC, 24-h urine free cortisol

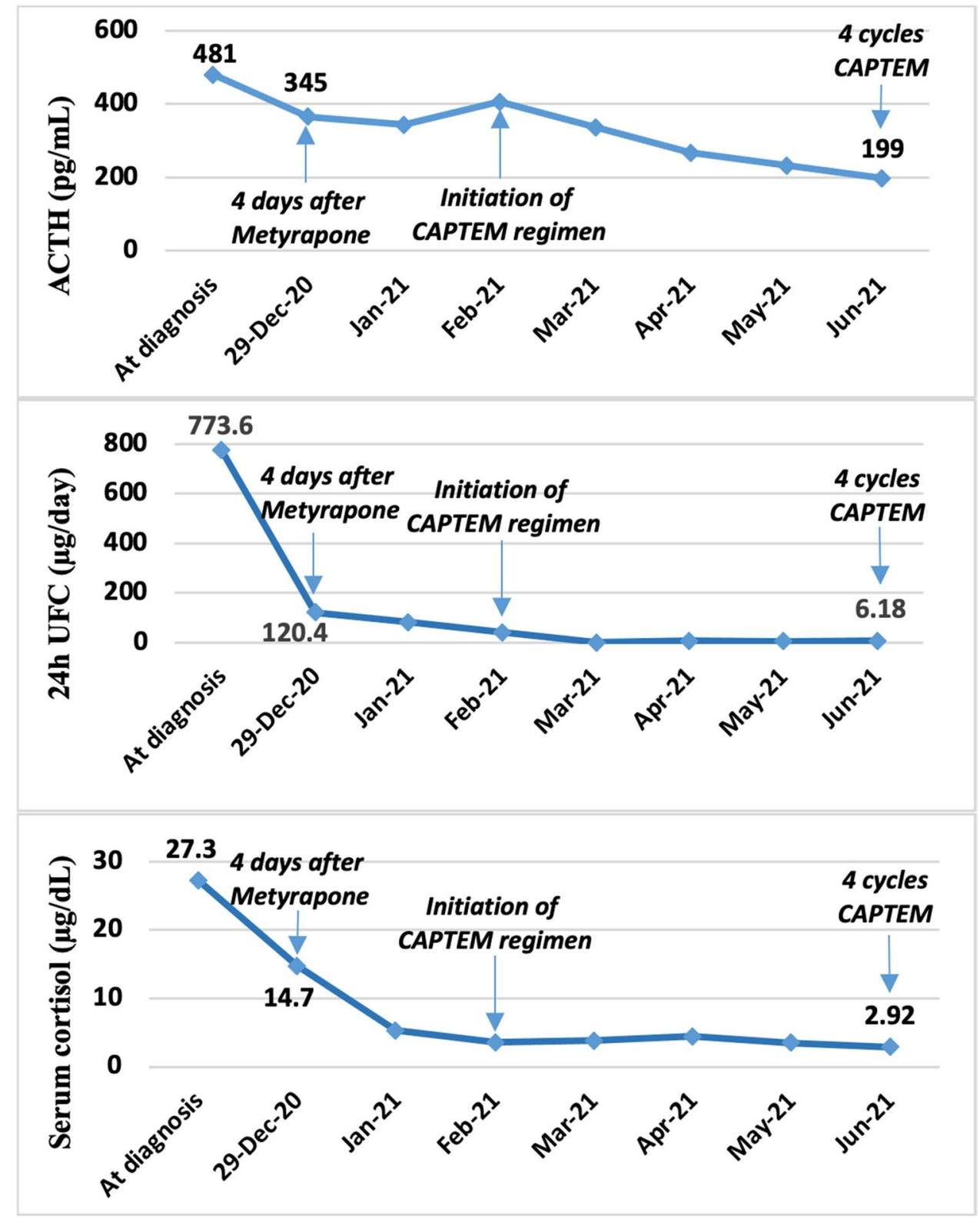

bone metastases were more extensive in ${ }^{18} \mathrm{FDG}$-PET/CT than ${ }^{68} \mathrm{Ga}$-SSTR PET/CT scan; however, the primary tumor could not be determined with either imaging method.

In the literature review, 35 cases of UPO NEN secreting ACTH have been previously reported [4, 18-26]. The female to male ratio of the 23 patients with available data was $13: 10$, and the age range of 10 of these patients was 30-55 years [18-21, 25]. The metastases were in the liver and abdominal cavity in the six cases whose metastatic sites were mentioned [4, 18, 19, 25]. However, in our case, the lesions confirming the diagnosis of NEN were in the mediastinal lymph nodes and bones. The absence of hepatic involvement may be attributed to the early presentation of the current case, whose primary source is probably not the gastroenteropancreatic region. In addition, bone metastases are more prevalent in pulmonary carcinoids, unlike digestive NENs [27].

In line with the recommendations of the European Neuroendocrine Tumor Society (ENETS) consensus report, in the context of metastatic presentation as in the current case, diffuse staining with TTF-1 and negative staining with CDX2, thyroglobulin, and calcitonin; the low Ki-67 labeling index; and the presence of two mitoses in $2 \mathrm{~mm}^{2}$ indicated that the origin was almost certainly an $\mathrm{AC}[3,27$, 28]. Locoregional and/or distant metastasis can be seen at the time of diagnosis in $15 \%$ of typical carcinoids and in about half of ACs with visible primaries [27]. The present case demonstrates that even ACs that cannot be visualized may present with metastatic disease. 
While hormonal risk is dominant in occult tumors secreting ACTH, tumoral risk is prominent in UPO NENs. However, in all cases, it is recommended to control hypercortisolemia and treat related comorbidities first and then perform a diagnostic evaluation. Moreover, thromboprophylaxis and Pneumocystis jirovecii pneumonia prophylaxis should be started [2]. The effectiveness of metyrapone (ranging from 500 to $3750 \mathrm{mg} /$ day) in controlling hypercortisolemia in both the short and the long term has been demonstrated in a retrospective analysis involving both CD and ECS patients [29]. In the current case, UFC levels were reduced to the normal range with the lowest dose of metyrapone $(500 \mathrm{mg} / \mathrm{day})$. When the symptoms of adrenal insufficiency appeared, hydrocortisone was added, and a "block and replace" regimen was applied as recommended [30]. In the 7-month follow-up period of our case, there was no additional problem other than controllable hypokalemia associated with metyrapone.

In the ENETS consensus report, it was emphasized that the treatment decision in UPO NEN cases should be made according to the tumor grade, functionality, somatostatin receptor (SSTR) status, spread of the tumor, and hepatic tumor burden [3]. In the current case, an advanced AC with a low proliferation index was hypothesized, and somatostatin analog (SSA) treatment and the CAPTEM regimen were initiated. In addition to the cytotoxic therapy, bone-specific treatments, including radiotherapy and anti-resorptive therapy, were applied due to the high tumor burden in the bones rather than because of the negative effects of CS.

SSA is recommended as first-line treatment in low proliferation index ACs with positive SSTR, but there is no clear consensus on the initial dose [27]. Randomized, placebocontrolled trials conducted in digestive NENs showed positive effects of octreotide LAR $30 \mathrm{mg}$ and lanreotide $120 \mathrm{mg}$ in tumor control [31, 32]. In the present case, after continued stabilization of hypercortisolemia and no disease progression detected on sequential imaging, SSA treatment was continued with an initial dose of $90 \mathrm{mg} / \mathrm{month}$ of lanreotide.

Although it is stated in the ENETS consensus report that the overall response rate is below $30 \%$ with current regimens in advanced ACs, it is recommended to administer cisplatin-based chemotherapy regimens in high proliferation index disease, and temozolomide alone or in combination or streptozotocin-based chemotherapy regimens in low proliferation index disease. mTOR inhibitors such as everolimus are recommended in advanced ACs after the failure of other treatments [27]. In a recent study, Al-Toubah et al. reported that by applying the CAPTEM regimen in 19 patients with advanced lung NENs, a partial response was obtained in $30 \%$ of the patients and stable disease in 55\%, while some patients had a tolerable toxicity profile, although adverse reactions were experienced [33]. In the present case, significant regression in metastatic lesions was observed 4 months after the initiation of cytotoxic treatment without any adverse reaction.

In conclusion, ECS is a rare entity that requires rapid management of hypercortisolemia because it may lead to fatal consequences and often involves long diagnostic processes. Past or active infections and incidental lesions may cause confusion and lead to many additional examinations. As in the present case, even radiologically invisible NENs with a relatively low Ki-67 labeling index and low mitosis count can be revealed with distant metastases at the time of diagnosis. After controlling hypercortisolemia in UPO NEN cases, it is important to characterize the possible primary origin to arrive at a treatment decision. Although the benefit of current chemotherapy regimens in metastatic disease is limited, cytotoxic treatment regimens combined with surgery and/or radiotherapy when necessary should be applied using a patient-based approach.

Author contributions H.B., H.D., and P.A. designed the article. H.B., H.D., P.A., M.C., T.T.T., A.K.E., and S.A.D. performed the assays and collected and interpreted the data. H.B. and H.D. wrote the initial manuscript. T.T.T., M.K., M.E.S., B.U., M.O., and E.C. reviewed the manuscript critically. All authors approved the final version of the manuscript and agree to be accountable for all aspects of the work.

\section{Declarations}

Informed consent Written informed consent was obtained from the patient before the study.

Conflict of interest The authors declare no competing interests.

Consent to publish Written informed consent was provided from the patient for publication of this case report and accompanying images.

\section{References}

1. Lacroix A, Feelders RA, Stratakis CA, Nieman LK (2015) Cushing's syndrome. Lancet 386:913-927. https://doi.org/10.1016/ S0140-6736(14)61375-1

2. Young J, Haissaguerre M, Viera-Pinto O, Chabre O, Baudin E, Tabarin A (2020) Management of endocrine disease: Cushing's syndrome due to ectopic ACTH secretion: an expert operational opinion. Eur J Endocrinol 182:R29-R58. https://doi.org/10.1530/ EJE-19-0877

3. Pavel M, Costa F, Capdevila J, Gross D, Kianmanesh R, Krenning E et al (2016) ENETS consensus guidelines update for the management of distant metastatic disease of intestinal, pancreatic, bronchial neuroendocrine neoplasms (NEN) and NEN of unknown primary site. Neuroendocrinology 103:172-185. https:// doi.org/10.1159/000443167

4. Lase I, Strele I, Grönberg M, Kozlovacki G, Welin S, Janson ET (2020) Multiple hormone secretion may indicate worse prognosis in patients with ectopic Cushing's syndrome. Hormones (Athens) 19:351-360. https://doi.org/10.1007/s42000-019-00163-z 
5. Kamp K, Alwani RA, Korpershoek E, Franssen GJH, de Herder WW, Feelders RA (2016) Prevalence and clinical features of the ectopic ACTH syndrome in patients with gastroenteropancreatic and thoracic neuroendocrine tumors. Eur J Endocrinol 174:271280. https://doi.org/10.1530/EJE-15-0968

6. Kaltsas GA, Besser GM, Grossman AB (2004) The diagnosis and medical management of advanced neuroendocrine tumors. Endocr Rev 25:458-511. https://doi.org/10.1210/er.2003-0014

7. Alexandraki K, Angelousi A, Boutzios G, Kyriakopoulos G, Rontogianni D, Kaltsas G (2017) Management of neuroendocrine tumors of unknown primary. Rev Endocr Metab Disord 18:423-431. https://doi.org/10.1007/s11154-017-9437-9

8. Wang Y-Z, Chauhan A, Rau J, Diebold AE, Opoku-Boateng A, Ramcharan T et al (2016) Neuroendocrine tumors (NETs) of unknown primary: is early surgical exploration and aggressive debulking justifiable? Chin Clin Oncol 5:4. https://doi.org/10. 3978/j.issn.2304-3865.2016.02.03

9. Wang SC, Parekh JR, Zuraek MB et al (2010) Identification of unknown primary tumors in patients with neuroendocrine liver metastases. Arch Surg 145:276-280. https://doi.org/10.1001/archs urg. 2010.10

10. Bhosale P, Shah A, Wei W et al (2013) Carcinoid tumours: predicting the location of the primary neoplasm based on the sites of metastases. Eur Radiol 23:400-407. https://doi.org/10.1007/ s00330-012-2615-y

11. Trott MJ, Farah G, Stokes VJ, Wang LM, Grossman AB (2016) A thymic neuroendocrine tumour in a young female: a rare cause of relapsing and remitting Cushing's syndrome. Endocrinol Diabetes Metab Case Rep 2016:160018. https://doi.org/10.1530/ EDM-16-0018

12. Oldfield EH, Doppman JL, Nieman LK et al (1991) Petrosal sinus sampling with and without corticotropin-releasing hormone for the differential diagnosis of Cushing's syndrome. N Engl J Med 325:897-905. https://doi.org/10.1056/NEJM199109263251301

13. Sookur PA, Sahdev A, Rockall AG et al (2009) Imaging in covert ectopic ACTH secretion: a CT pictorial review. Eur Radiol 19:1069-1078. https://doi.org/10.1007/s00330-008-1274-5

14. Zemskova MS, Gundabolu B, Sinaii N et al (2010) Utility of various functional and anatomic imaging modalities for detection of ectopic adrenocorticotropin-secreting tumors. J Clin Endocrinol Metab 95:1207-1219. https://doi.org/10.1210/jc.2009-2282

15. Gabriel S, Garrigue P, Dahan L et al (2018) Prospective evaluation of 68Ga-DOTATATE PET/CT in limited disease neuroendocrine tumours and/or elevated serum neuroendocrine biomarkers. Clin Endocrinol (Oxf) 89:155-163. https://doi.org/10.1111/cen.13745

16. Moore W, Freiberg E, Bishawi M et al (2013) FDG-PET imaging in patients with pulmonary carcinoid tumor. Clin Nucl Med 38:501-505. https://doi.org/10.1097/RLU.0b013e318279f0f5

17. Panagiotidis E, Alshammari A, Michopoulou S et al (2017) Comparison of the impact of 68Ga-DOTATATE and 18F-FDG $\mathrm{PET} / \mathrm{CT}$ on clinical management in patients with neuroendocrine tumors. J Nucl Med 58:91-96. https://doi.org/10.2967/jnumed. 116.178095

18. Kornely E, Schlaghecke R, Horster FA (1991) Palliative therapy of an ectopic cushing's syndrome due to a metastatic carcinoid tumor. Klin Wochenschr 69:173-176. https://doi.org/10.1007/ BF01665863

19. Takami H, Ogino Y, Tanaka K, Kubo A (1998) Somatostatinreceptor-negative carcinoid tumour responsible for Cushing's syndrome. Eur J Surg Oncol 24:337-338. https://doi.org/10.1016/ s0748-7983(98)80022-x

20. Aniszewski JP, Young WF Jr, Thompson GB, Grant CS, van Heerden JA (2001) Cushing syndrome doe to ectopic adrenocorticotropic hormone secretion. World J Surg 25:934-940. https:// doi.org/10.1007/s00268-001-0032-5
21. Ilias I, Torpy DJ, Pacak K, Mullen N, Wesley RA, Nieman LK (2005) Cushing's syndrome due to ectopic corticotropin secretion: twenty years' experience at the National Institutes of Health. J Clin Endocrinol Metab 90:4955-4962. https://doi.org/10.1210/ jc. 2004-2527

22. Isidori AM, Kaltsas GA, Pozza C et al (2006) The ectopic adrenocorticotropin syndrome: clinical features, diagnosis, management, and long-term follow-up. J Clin Endocrinol Metab 91:371-377. https://doi.org/10.1210/jc.2005-1542

23. Ejaz S, Vassilopoulou-Sellin R, Busaidy NL et al (2011) Cushing syndrome secondary to ectopic adrenocorticotropic hormone secretion: the University of Texas MD Anderson Cancer Center Experience. Cancer 117:4381-4389. https://doi.org/10.1002/cncr. 26029

24. Daskalakis K, Chatzelis E, Tsoli M et al (2019) Endocrine paraneoplastic syndromes in patients with neuroendocrine neoplasms. Endocrine 64:384-392. https://doi.org/10.1007/ s12020-018-1773-3

25. Albani A, Berr CM, Beuschlein F et al (2019) A pitfall of bilateral inferior petrosal sinus sampling in cyclic Cushing's syndrome. BMC Endocr Disord 19:105. https://doi.org/10.1186/ s12902-019-0433-9

26. Wannachalee T, Turcu AF, Bancos I et al (2019) The clinical impact of [68Ga]-DOTATATE PET/CT for the diagnosis and management of ectopic adrenocorticotropic hormone-secreting tumours. Clin Endocrinol (Oxf) 91:288-294. https://doi.org/10. 1111/cen.14008

27. Caplin ME, Baudin E, Ferolla P et al (2015) Pulmonary neuroendocrine (carcinoid) tumors: European Neuroendocrine Tumor Society expert consensus and recommendations for best practice for typical and atypical pulmonary carcinoids. Ann Oncol 26:1604-1620. https://doi.org/10.1093/annonc/mdv041

28. Kim JY, Hong S-M, Ro JY (2017) Recent updates on grading and classification of neuroendocrine tumors. Ann Diagn Pathol 29:11-16. https://doi.org/10.1016/j.anndiagpath.2017.04.00

29. Daniel E, Aylwin S, Mustafa O et al (2015) Effectiveness of metyrapone in treating Cushing's syndrome: a retrospective multicenter study in 195 patients. J Clin Endocrinol Metab 100:41464154. https://doi.org/10.1210/jc.2015-2616

30. Corcuff J-B, Young J, Masquefa-Giraud P, Chanson P, Baudin E, Tabarin A (2015) Rapid control of severe neoplastic hypercortisolism with metyrapone and ketoconazole. Eur J Endocrinol 172:473-481. https://doi.org/10.1530/EJE-14-0913

31. Rinke A, Muller HH, Schade-Brittinger C et al (2009) Placebocontrolled, double-blind, prospective, randomized study on the effect of octreotide LAR in the control of tumor growth in patients with metastatic neuroendocrine midgut tumors: a report from the PROMID Study Group. J Clin Oncol 27:4656-4663. https://doi. org/10.1200/JCO.2009.22.8510

32. Caplin ME, Pavel M, Ćwikła JB et al (2016) Anti-tumour effects of lanreotide for pancreatic and intestinal neuroendocrine tumours: the CLARINET open-label extension study. Endocr Relat Cancer 23:191-199. https://doi.org/10.1530/ERC-15-0490

33. Al-Toubah T, Morse B, Strosberg J (2020) Capecitabine and temozolomide in advanced lung neuroendocrine neoplasms. Oncologist 25:e48-e52. https://doi.org/10.1634/theoncologist.2019-0361

Publisher's note Springer Nature remains neutral with regard to jurisdictional claims in published maps and institutional affiliations. 